\title{
Synthesis and Characterization of Novel Water Soluble Starch Tartarate Nanoparticles
}

\author{
Suk Fun Chin, Suh Cem Pang, and Lih Shan Lim \\ Department of Chemistry, Faculty of Resource Science and Technology, Universiti Malaysia Sarawak, \\ 94300 Kota Samarahan, Malaysia \\ Correspondence should be addressed to Suk Fun Chin, sukfunchin@gmail.com
}

Received 10 November 2011; Accepted 30 November 2011

Academic Editor: S. Matsuya

Copyright @ 2012 Suk Fun Chin et al. This is an open access article distributed under the Creative Commons Attribution License, which permits unrestricted use, distribution, and reproduction in any medium, provided the original work is properly cited.

Starch tartarate nanoparticles were prepared by esterification reaction between native sago starch (Metroxylon sagu) and tartaric acid using dimethyl sulphoxide (DMSO) as a solvent and 2-dimethylamino pyridine (DMAP) as a catalyst at $100^{\circ} \mathrm{C}$. The substitution of tartaric acid onto native sago starch was confirmed by the FTIR spectra which showed the presence of carbonyl group absorption band of tartarate ester. The solubility of the as-synthesized starch tartarate nanoparticles was observed to increase linearly with increase in the degrees of substitution. Under optimized synthesis conditions, starch tartarate nanoparticles of mean particle sizes which ranged from $200 \mathrm{~nm}$ to $300 \mathrm{~nm}$ were produced.

\section{Introduction}

Starch as a polysaccharide is one of the most abundant renewable agroresources. Starch mainly composed of amylose and amylopectin polysaccharides. The ratio of amylopectin and amylose depends on the plant origin such as maize, corn, sago, tapioca, wheat, and potato $[1,2]$. Starch has been traditionally being widely used as a major low-cost food item in many countries. Due to its low-toxicity, abundant availability, biodegradable, and low cost, starch and its derivatives are widely used in various industry applications such as coating and sizing in paper industries, binder and adhesives, absorbent, drug delivery carriers, and implants [3-6]. However, the inherent poor mechanical properties, insolubility in water, and high viscosity have limited the utilization of native starch in various applications. This drawback has prompted many researchers to modify starch in order to improve its properties for various technological and biomedical applications. The properties of modified starch were affected by several factors, such as the degree of substitution (DS), sources of starch, and types of substituent [7].

The esterification reaction is one of the most commonly used approaches to modify starch. Among the commonly studied starch esters are starch acetate $[8,9]$ and starch maleate [10]. Starch esters are being used in applications such as substitutions for petroleum-based plastic materials, sealing adhesives, and biodegradable packing materials [11]. Various synthesis methods have been used to synthesize starch esters. The esterification of starch is normally carried out by reacting starch with fatty acid chlorides [12], acetic anhydrides [13], succinic anhydrides [14], sodium selenite [15], or dicarboxylic acid [16] in various types solvents and under basic conditions.

In this work, we have attempted to prepare a new type of water soluble starch esters by the esterification reaction between native sago starch and tartaric acid. Tartaric acid is a nontoxic, natural occurring compound found in fruits such as grapes, bananas, and tamarinds. Tartaric acid is being used as a popular food additive in soft drink, cream of tartar and baking powder. Being a dicarboxylic acid, the esterification of starch can occur through the substitution of carboxylic group of the tartaric acid onto the free hydroxyl groups of starch molecules.

\section{Experimental}

2.1. Materials. Native sago (Metroxylon sagu) starch was purchased from a local grocery store. Ultrapure water $(\sim 18.2 \mathrm{M} \Omega)$ was obtained from Water Purifying System 

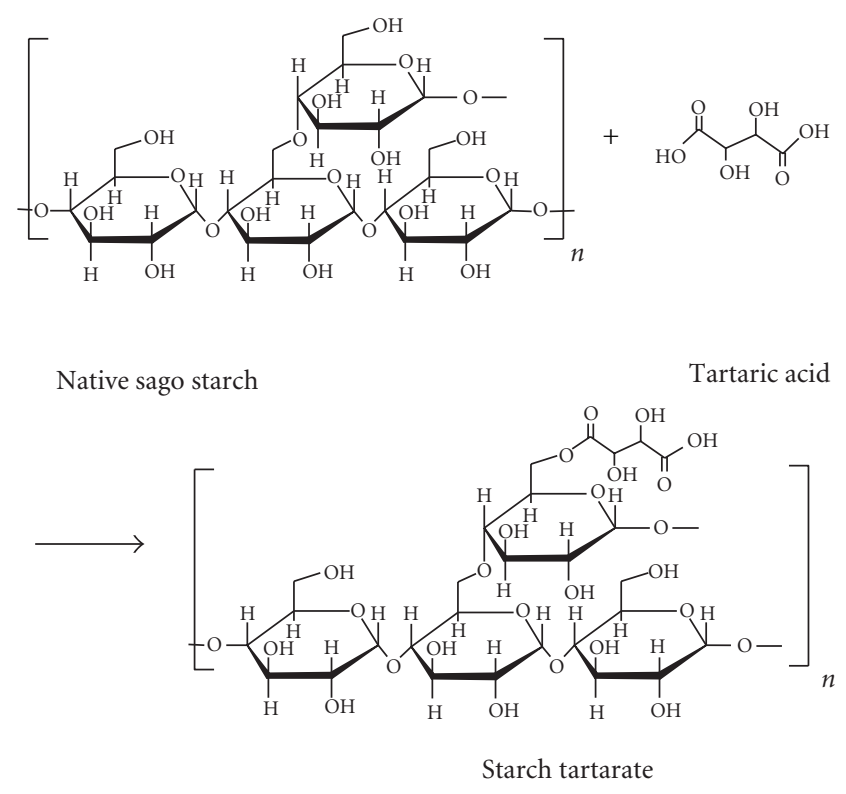

Scheme 1: Esterification reaction of native sago starch and tartaric acid.

(ELGA, Model Ultra Genetic). All the chemicals were of reagent grade and were used without further purification.

\subsection{Preparation of Starch Tartarate Nanoparticles. The syn-} thesis of starch tartarate nanoparticles was carried out by the esterification reaction between free hydroxyl groups of starch and carboxylic groups of tartaric acid (Scheme 1).

In a typical synthesis, $0.81 \mathrm{~g}(0.005 \mathrm{~mol}$ of Anhydrous Glucose Unit (AGU)) of native sago starch was added into $5 \mathrm{~mL}$ of DMSO under magnetically stirring. $0.1 \mathrm{~mL}$ of 2(dimethylamino) pyridine (DMAP) was added as a catalyst to the starch mixture. Tartaric acid dissolved in $5 \mathrm{~mL}$ DMSO was then added dropwise to the starch mixture. The starch mixture was magnetically stirred and heated to $100^{\circ} \mathrm{C}$ for various reaction durations. The reaction mixture was then precipitated in absolute ethanol and rinsed twice with absolute ethanol to remove excess of tartaric acid, DMSO, and water. The precipitated starch tartarate nanoparticles were separated from the solvent by centrifugation and dried at $60^{\circ} \mathrm{C}$ in oven for 24 hours.

\subsection{Characterization of Starch Tartarate}

2.3.1. Scanning Electron Microscopy (SEM). The morphology of starch tartarate nanoparticles was observed using a scanning electron microscope (SEM) (JEOL Model JSM 6390LA) at various magnifications.

2.3.2. Fourier Transform Infrared Spectroscopy (FTIR). FTIR spectra of the samples were obtained using a Fourier Transformed Infrared Spectrometer (SHIMADZU Model FTIR8201PC). Dried starch tartarate powder was made into thin pellets with potassium bromide $(\mathrm{KBr})$ and scanned with an FT-IR spectrometer within the wave number ranges of 400 and $4000 \mathrm{~cm}^{-1}$.
2.3.3. Degree of Substitution (DS). The degree of substitution (DS) was determined using the titration method reported by Ogawa et al. with modifications [17]. $0.05 \mathrm{~g}$ of dried starch tartarate was dissolved in $10 \mathrm{~mL}$ of $0.1 \mathrm{M} \mathrm{NaOH}$ by stirring at room temperature for 30 minutes. Excess $\mathrm{NaOH}$ in the solution was then titrated with $0.1 \mathrm{M}$ hydrochloric acid $(\mathrm{HCl})$ solution until the solution was neutral. DS was calculated using the following formula (2):

Percentage of substitution, Ta\%

$$
\begin{aligned}
& =\frac{M_{\mathrm{NaOH}} \times V_{\mathrm{HCl}} \times \mathrm{Mw}_{\mathrm{Ta}}}{W_{\mathrm{ST}}}, \\
\mathrm{DS} & =\frac{162 \times \mathrm{Ta} \%}{13300-(132 \times \mathrm{Ta} \%)},
\end{aligned}
$$

where $M_{\mathrm{NaOH}}$ is the molarity of $\mathrm{NaOH}, V_{\mathrm{HCl}}$ is the volume $(\mathrm{mL})$ of $\mathrm{HCl}$ used, $W_{\mathrm{ST}}$ is the weight of starch tartarate, and $\mathrm{Mw}_{\mathrm{Ta}}$ is the molecular weight of tartaric acid substituents $\left[\mathrm{COOH}(\mathrm{CHOH})_{2} \mathrm{CO}-\right]$ equal to 133.

2.3.4. Solubility of Starch Tartarate. The solubility of starch tartarate in water was studied at different temperatures and $\mathrm{pH}$ values. The dried starch tartarate samples were weighed and immersed in $50 \mathrm{~mL}$ of water for 24 hours. The swollen samples were then dried in an oven at $60^{\circ} \mathrm{C}$ for 24 hours. The dried samples were then weighed until a constant weigh was obtained. The percentage of solubility of the starch tartarate, was calculated according to (3):

Percentage of solubility

$$
=\frac{\text { weight of initial sample }- \text { dried sample }}{\text { weight of initial sample }} \times 100 \text {. }
$$




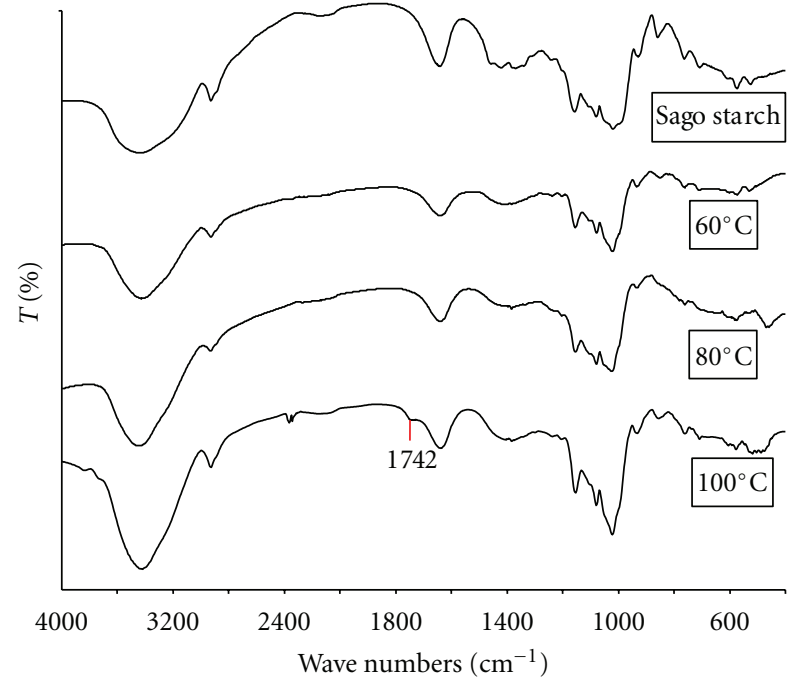

FIGURE 1: FTIR spectra of samples at various reaction temperatures.

\section{Results and Discussion}

3.1. Effect of Reaction Temperatures. The effect of reaction temperatures on the esterification reaction was investigated by heating samples at various reaction temperatures. FTIR spectra of samples prepared at various reaction temperatures were shown in Figure 1. As shown in Figure 1, the characteristic peak of carbonyl functional group at around $1740 \mathrm{~cm}^{-1}$ was only observed for sample prepared at $100^{\circ} \mathrm{C}$. This suggested that the formation of ester bond of $\mathrm{C}=\mathrm{O}$ by the esterification reaction between starch molecules and tartaric acid to form starch tartarate only occurred at $100^{\circ} \mathrm{C}$. At reaction temperature lower than $100^{\circ} \mathrm{C}$, no esterification reaction occurred. In addition, DMAP as a catalyst was required for this esterification reaction to occur.

3.2. Effect of Reaction Durations. Figure 2 shows FTIR spectra of samples prepared at various reaction durations. The characteristic peak of carbonyl functional group was only observed for samples obtained from reactions that were carried out for more than 4 hours. The intensity of the carbonyl group at around $1740 \mathrm{~cm}^{-1}$ was observed to increase with reaction duration from 4 to 8 hours. The increase in intensity of the characteristic peak of carbonyl group demonstrated that higher degree of substitution of hydroxyl groups $(\mathrm{OH})$ of starch molecules with carboxylic groups $(\mathrm{COOH})$ from tartaric acid was achieved through longer reaction duration. However, reaction duration longer than 6 hours was not pursued in the current study as prolonged reaction duration ( $>6$ hours) was observed to cause degradation of starch.

3.3. Degree of Substitution and Solubility. The degree of substitution (DS) was calculated as the average number of hydroxyl groups substituted per AGU. As shown in Figure 3, the degree of substitution was observed to increase with longer reaction duration from 2 to 8 hours. This finding concurs with observation of increased intensity of characteristic peak

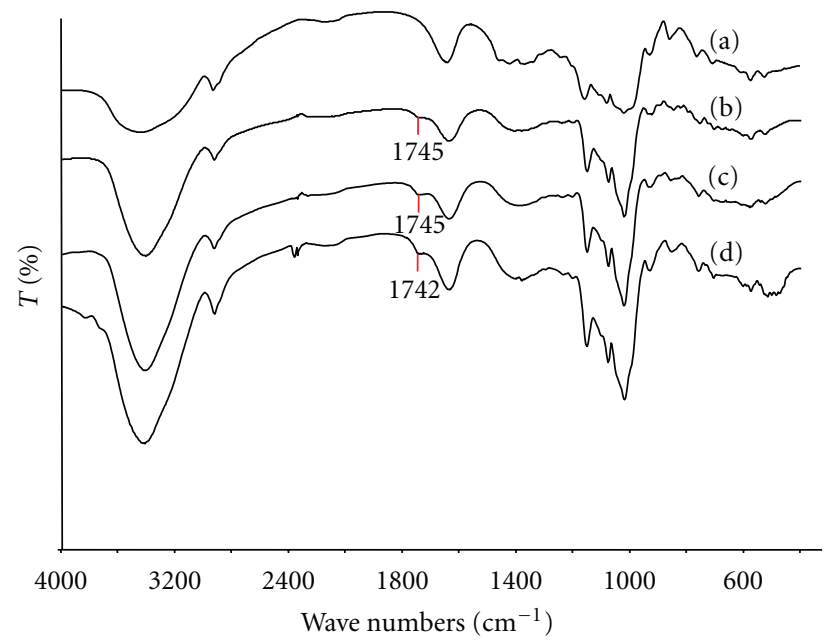

FIGURE 2: FTIR spectra of samples at various reaction duration: (a) 0 hour, (b) 2 hours, (c) 4 hours, and (d) 6 hours.

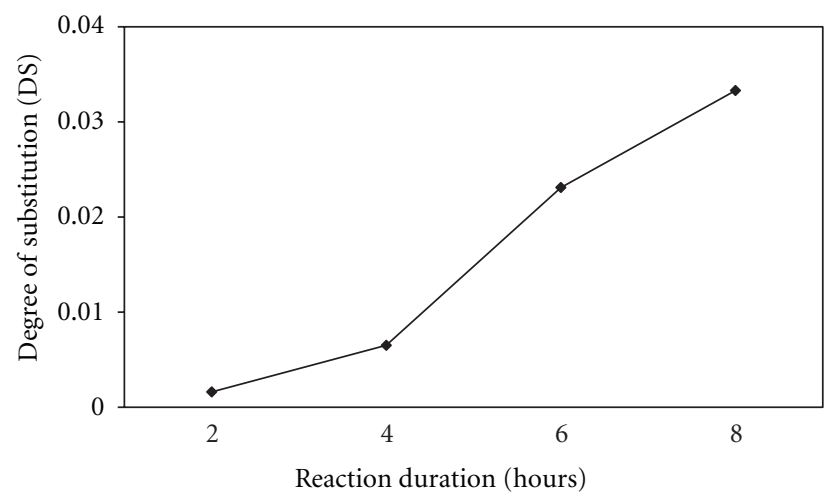

FIGURE 3: Effects of reaction duration on the degree of substitution of starch tartarate.

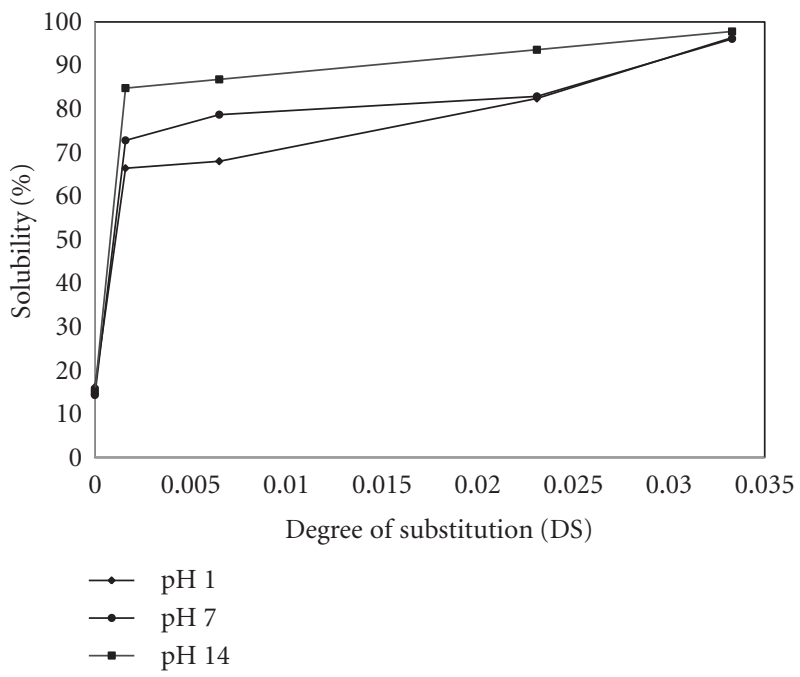

FIGURE 4: Effect of degree of substitution on the solubility of starch tartarate at various $\mathrm{pH}$ values. 


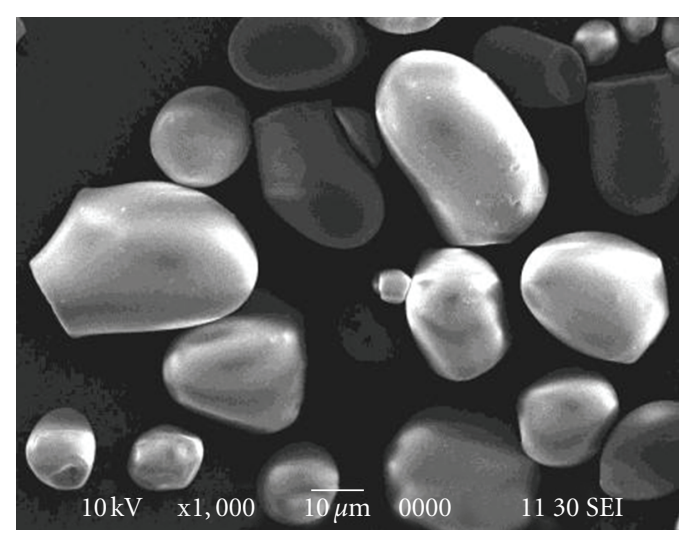

(a)

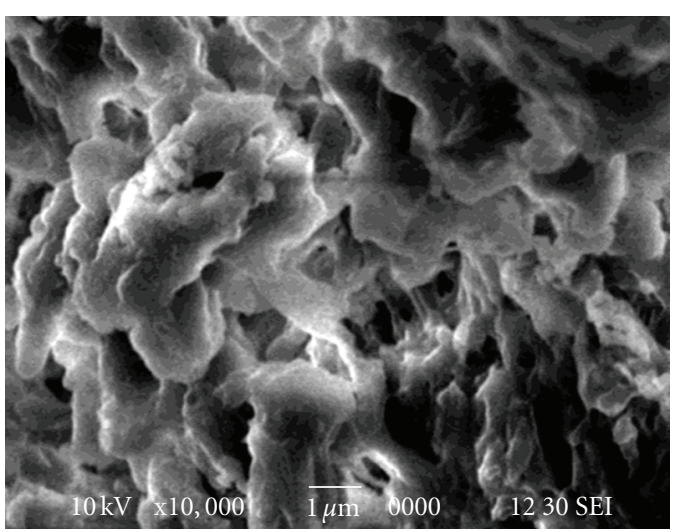

(b)

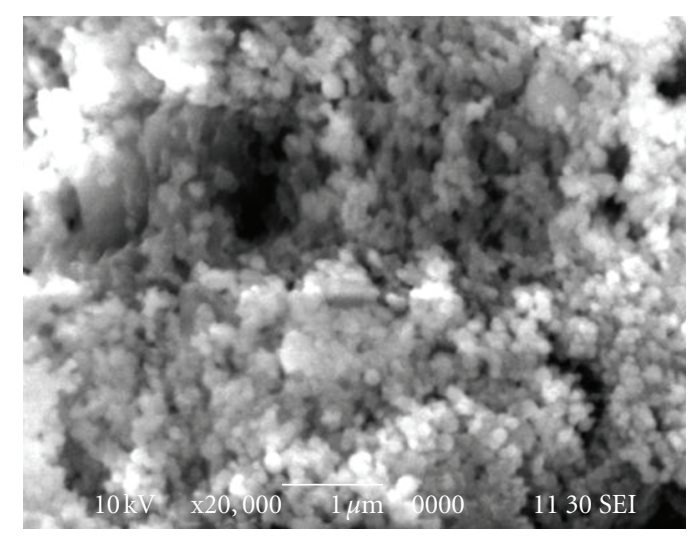

(c)

FIGURE 5: SEM images of (a) native sago starch, (b) regenerated native sago starch, and (c) starch tartarate nanoparticles.

of carbonyl group at around $1740 \mathrm{~cm}^{-1}$ as observed in FTIR spectra (Figure 2). As the reaction time was longer, there is more time for the $-\mathrm{COOH}$ groups of tartaric acid to react with the $-\mathrm{OH}$ groups of starch molecules and thus more substitution could occur.

Increase in the degree of substitution was observed to enhance the solubility of tartaric acid modified starch in water at various $\mathrm{pH}$ values as shown in Figure 4. As more hydroxyl groups per AGU of starch molecules were substituted with tartaric acid, the free carboxylic functional groups of tartaric acid ionize in solution to form carboxylate ions $\left(\mathrm{COO}^{-}\right)$and resulted in the solubility of starch tartarate in water. The solubility of starch-tartarate in water was observed to increase under basic conditions ( $\mathrm{pH} 14)$ as compared to neutral $(\mathrm{pH} 7)$ and acidic $(\mathrm{pH} \mathrm{1)}$ conditions. Under basic condition, starch tartarate ionizes more easily and thus is more soluble in water. However, at higher degree of substation, starch tartarate was observed to dissolve easily in water regardless of the $\mathrm{pH}$ values of the solution. The good water solubility of starch tartarate was due to the high coverage of carboxylic acid that can be easily ionized in water.

3.4. Surface Morphology. The native sago starch granules in Figure 5(a) were observed to be smooth and oval in shape. The sizes of the starch granules varied between 6 and $49 \mu \mathrm{m}$ in diameters. Native sago starch was first dissolved in DMSO solvent and reprecipitated using absolute ethanol. As can be seen from Figure 5(b), the structure of the native sago starch had collapsed during their dissolution and regeneration process to form homogenous agglomerates. After the native sago starch was modified with tartaric acid, the resulting starch tartarate precipitated in absolute alcohol was observed to form spherical nanoparticles with size ranged from 200 to $300 \mathrm{~nm}$. Starch tartarate nanoparticles were formed by the desolvation of starch tartarate due to the rapid gradientdriven diffusion of solvents into the nonsolvent $[18,19]$.

\section{Conclusion}

In conclusion, we have successfully synthesized water soluble starch tartarate nanoparticles by reacting starch and tartaric acid with DMSO as a solvent and DMAP as a catalyst. Starch tartarate of moderate degree of substitution (0.0230.033 ) was synthesized from the esterification reaction. Water soluble starch tartarate nanoparticles with size ranged from 200 to $300 \mathrm{~nm}$ were produced by precipitation of starch tartarate in absolute ethanol. These water soluble starch tartarate nanoparticles are promising materials for various biomedical applications such as drug delivery carriers and artificial implants. 


\section{Acknowledgment}

Financial support by Malaysia Ministry of Higher Education (MOHE) Fundamental Research Grant Scheme (FRGS), Grant no. 01(17)746/2010(32) was gratefully acknowledged.

\section{References}

[1] J. Y. Li and A. I. Yeh, "Relationships between thermal, rheological characteristics and swelling power for various starches," Journal of Food Engineering, vol. 50, no. 3, pp. 141-148, 2001.

[2] T. Aberle, W. Burchard, W. Vorwerg, and S. Radosta, "Conformational contributions of amylose and amylopectin to the structural properties of starches from various sources," Starch, vol. 46, no. 9, pp. 329-335, 1994.

[3] J. Lörcks, "Properties and applications of compostable starchbased plastic material," Polymer Degradation and Stability, vol. 59, no. 1-3, pp. 245-249, 1998.

[4] Q. Fang and M. A. Hanna, "Functional properties of polylactic acid starch-based loose-fill packaging foams," Cereal Chemistry, vol. 77, no. 6, pp. 779-783, 2000.

[5] A. Cova, A. J. Sandoval, V. Balsamo, and A. J. Müller, "The effect of hydrophobic modifications on the adsorption isotherms of cassava starch," Carbohydrate Polymers, vol. 81, no. 3, pp. 660-667, 2010.

[6] Y. Dumoulin, S. Alex, P. Szabo, L. Cartilier, and M. A. Mateescu, "Cross-linked amylose as matrix for drug controlled release. X-ray and FT-IR structural analysis," Carbohydrate Polymers, vol. 37, no. 4, pp. 361-370, 1998.

[7] Z. Chen, H. A. Schols, and A. G. J. Voragen, "Differently sized granules from acetylated potato and sweet potato starches differ in the acetyl substitution pattern of their amylose populations," Carbohydrate Polymers, vol. 56, no. 2, pp. 219226, 2004.

[8] A. Biswas, R. L. Shogren, and J. L. Willett, "Solvent-free process to esterify polysaccharides," Biomacromolecules, vol. 6, no. 4, pp. 1843-1845, 2005.

[9] S. C. Pang, S. F. Chin, S. H. Tay, and F. M. Tchong, "Starchmaleate-polyvinyl alcohol hydrogels with controllable swelling behaviors," Carbohydrate Polymers, vol. 84, no. 1, pp. 424-429, 2011.

[10] A. Lehmann and B. Volkert, "Preparing esters from highamylose starch using ionic liquids as catalysts," Carbohydrate Polymers, vol. 83, no. 4, pp. 1529-1533, 2011.

[11] J. F. Mano, D. Koniarova, and R. L. Reis, “Thermal properties of thermoplastic starch/synthetic polymer blends with potential biomedical applicability," Journal of Materials Science, vol. 14, no. 2, pp. 127-135, 2003.

[12] A. Jorge, A. Isabelle, and B. Elisabeth, "Preparation of longchain esters of starch using fatty acid chlorides in the absence of an organic solvent," Starch, vol. 51, no. 4, pp. 132-135, 1999.

[13] B. Volkert, A. Lehmann, T. Greco, and M. H. Nejad, "A comparison of different synthesis routes for starch acetates and the resulting mechanical properties," Carbohydrate Polymers, vol. 79, no. 3, pp. 571-577, 2010.

[14] T. Yoshimura, R. Yoshimura, C. Seki, and R. Fujioka, "Synthesis and characterization of biodegradable hydrogels based on starch and succinic anhydride," Carbohydrate Polymers, vol. 64, no. 2, pp. 345-349, 2006.

[15] H. Staroszczyk, P. Tomasik, P. Janas, and A. Poreda, "Esterification of starch with sodium selenite and selenate," Carbohydrate Polymers, vol. 69, no. 2, pp. 299-304, 2007.
[16] J. K. John and K. C. M. Raja, "Properties of cassava starchdicarboxylic acid complexes," Carbohydrate Polymers, vol. 39, no. 2, pp. 181-186, 1999.

[17] K. Ogawa, I. Hirai, C. Shimasaki et al., "Simple determination method of degree of substitution for starch acetate," Bulletin of the Chemical Society of Japan, vol. 72, no. 12, pp. 2785-2790, 1999.

[18] S. Hornig, T. Heinze, C. R. Becer, and U. S. Schubert, "Synthetic polymeric nanoparticles by nanoprecipitation," Journal of Materials Chemistry, vol. 19, no. 23, pp. 3838-3840, 2009.

[19] U. Bilati, E. Allémann, and E. Doelker, "Development of a nanoprecipitation method intended for the entrapment of hydrophilic drugs into nanoparticles," European Journal of Pharmaceutical Sciences, vol. 24, no. 1, pp. 67-75, 2005. 

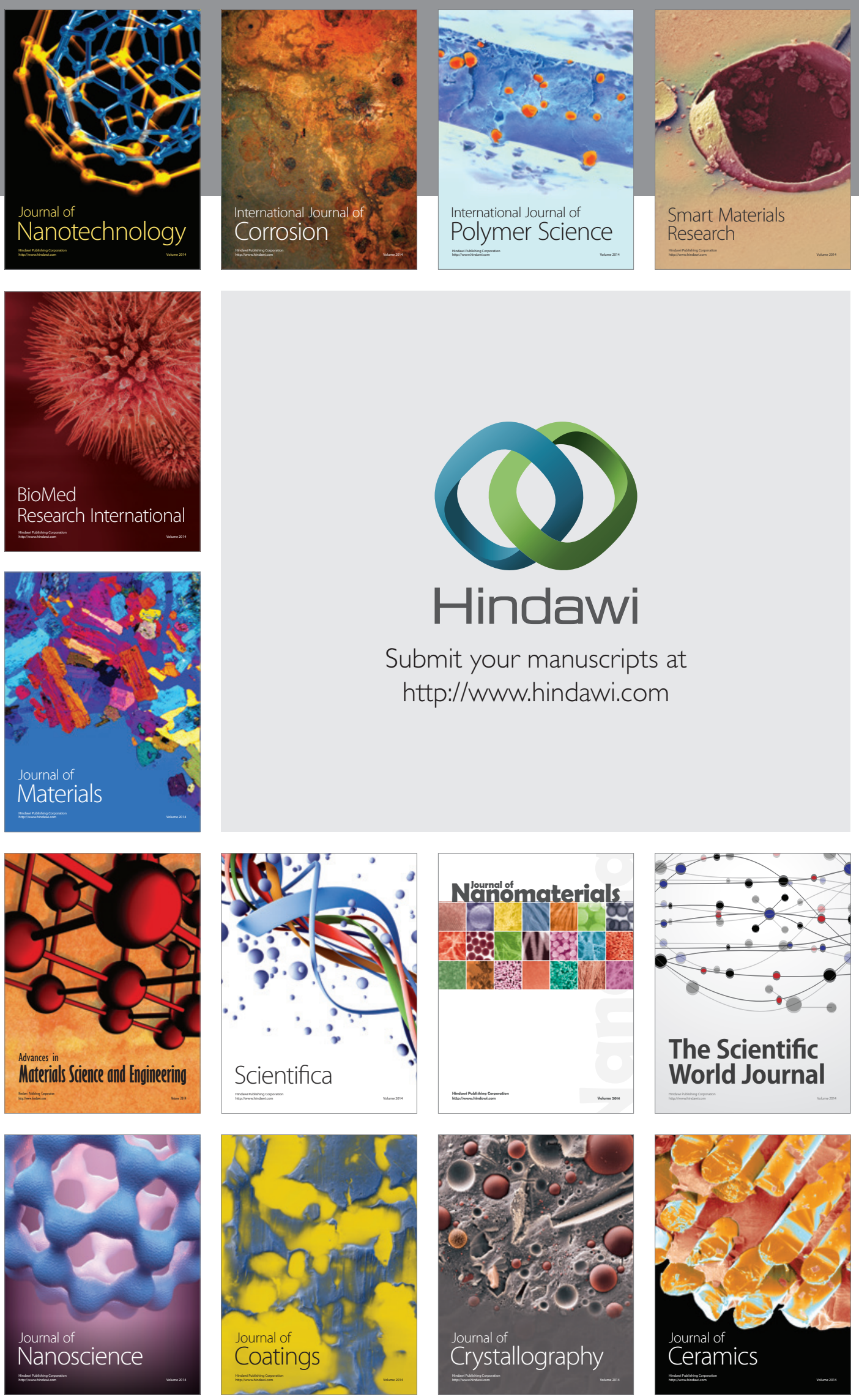

The Scientific World Journal

Submit your manuscripts at

http://www.hindawi.com

\section{World Journal}

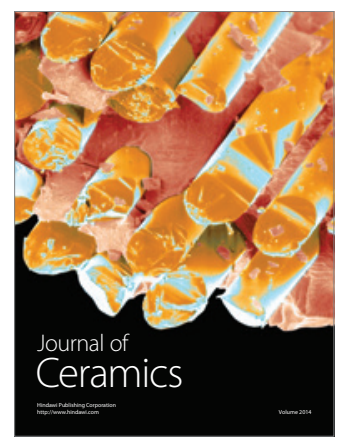

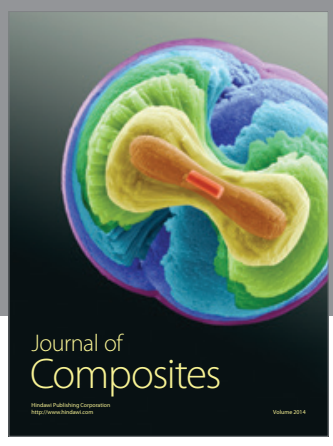
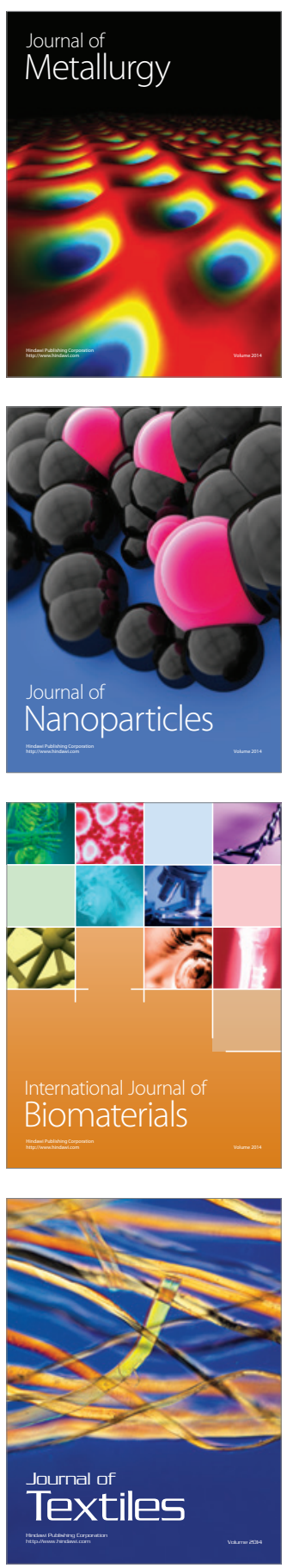